\title{
INTOXICAÇÕES EXÓGENAS NO MUNICÍPIO DE SANTARÉM-PARÁ NOS ANOS DE 2009 A 2013
}

\author{
Karen Cristini Yumi Ogawa MAESTRI ${ }^{1}$ \\ Alciene Ferreira da Silva VIANA2 \\ Aline Maria da Cruz LIMA ${ }^{3}$ \\ Dione Cristina Gemaque Batista de AGUIAR $^{4}$ \\ Andrew Mairom NOGUEIRA ${ }^{5}$ \\ Paulo Marcelo Pedroso PEREIRA ${ }^{6}$ \\ Aline GLASER ${ }^{7}$ \\ Régis Piloni MAESTRI ${ }^{8 *}$
}

1 - Mestre em Doenças Tropicais, Farmacêutica e docente do Instituto Esperança de Ensino Superior - IESPES, karenmaestri@ hotmail.com.br

${ }^{2}$ - Graduada em Farmácia pelo Instituto Esperança de Ensino Superior - IESPES, alcienev@ hotmail.com

3 - Graduada em Farmácia pelo Instituto Esperança de Ensino Superior - IESPES, alinemdcl@ hotmail.com

${ }^{4}$ _ Graduada em Farmácia pelo Instituto Esperança de Ensino Superior - IESPES, aguiardcgb@ hotmail.com

5 - Graduada em Farmácio pelo Instituto Esperança de Ensino Superior - IESPES, andrewmn @ hotmail.com

6 - Mestre em Docência, Matematico e docente do Instituto Esperança de Ensino Superior - IESPES paulommarcelo@hotmail.com

7. Enfermeira, Centro de Controle de Zoonoses, DIVISA, Santarém Pará, alinegss@ hotmail.com

$8^{*}$ - Mestre em Doenças Tropicais, Farmacêutico e docente do Instituto Esperança de Ensino Superior - IESPES regismaestri@hotmail.com

Recebido em: 03/11/2015 - Aprovado em: 17/04/2016 - Disponibilizado em: 30/07/2016

\section{RESUMO}

A intoxicação é um processo que pode ser causado por substâncias endógenas ou exógenas, caracterizados por desequilíbrio fisiológico, consequente das alterações bioquímicas no organismo em que é evidenciado por sinais e sintomas ou mediante dados laboratoriais. Com a diversidade dos produtos químicos ocorreu um aumento das intoxicações, ocasionando reflexos na saúde pública e ambiental. As intoxicações por agentes químicos é um problema de grande relevância para saúde pública e no Brasil são os principais responsáveis por intoxicações humanas. Esta pesquisa objetivou descrever o perfil das intoxicações no município de Santarém-Pará nos anos de 2009 a 2013. Neste estudo epidemiológico foram analisados 269 casos registrados no SINAN. As variáveis utilizadas foram: faixa etária; gênero; agente tóxico; zona de residência, tipo de exposição; critério de confirmação e evolução. Constatou-se que a intoxicação por medicamento representou 23,1\% (62/269) das intoxicações, seguida dos raticidas 19,7\% (53/269) dos casos, sendo a tentativa de suicídio a principal circunstância responsável. O sexo feminino apresentou o maior número de casos registrados, bem como os adultos jovens de 20 a 39 anos. Este estudo alerta para a continuidade das intoxicações, e para a necessidade de ações educativas e medidas de prevenção efetivas, pois a possibilidade de conhecimento da real dimensão do problema das intoxicações exógenas por meio de intensificação das notificações permitirá aos profissionais elegerem medidas de intervenções preventivas apropriadas para evitar a ocorrência desses eventos.

Palavras -Chave: Intoxicação. Agente tóxico.Toxicologia.Santarém. Epidemiologia. 


\title{
POISON EXOGENOUS NO SANTARÉM-PARA COUNTY IN THE YEARS 2009 TO 2013
}

\begin{abstract}
Poisoning is a process that can be caused by endogenous or exogenous substances, characterized by physiological imbalance, resulting from biochemical changes in the body in which is shown by signs and symptoms or laboratory data through. With the diversity of chemical poisoning was increased, causing effects on the environment and public health. Poisoning by chemicals is a very important problem for public health and Brazil are the main drivers of human poisoning. This study aimed to describe the profile of poisonings in the city of Santarem-Pará in 2009 to 2013 . In this epidemiological study analyzed 269 cases reported in SINAN. The variables used were: age; gender; toxic agent; area of residence, type of exposure; criteria for confirmation and evolution. It was found that the poisoning drug accounted for $23.1 \%(62 / 269)$ of poisonings, followed by rodenticides $19.7 \%(53 / 269)$ of the cases, attempted suicide the main condition responsible. The women had the highest number of registered cases, as well as young adults 20-39 years. This study points to the continuity of poisonings, and the need for educational and effective prevention measures, for the possibility to understand the real extent of the problem of exogenous intoxication through intensification of notifications allow professionals elect appropriate preventive interventions measures to prevent the occurrence of such events.
\end{abstract}

Keywords: Poisoning. Toxic agent.Toxicology. Santarém. Epidemiology.

\section{INTRODUÇÃO}

A intoxicação é um processo que pode ser causado por substâncias endógenas e exógenas. As substâncias endógenas são aquelas produzidas no próprio organismo e são purificadas pelo fígado e eliminadas pelos rins, quando estas são produzidas em excesso ou ocorrem um comprometimento na eliminação, resultará em intoxicação. As substâncias exógenas são aquelas substâncias nocivas que "vem de fora", podendo ocorrer por ingestão, por inalação ou por contato direto sobre várias partes do corpo (OGA, 2008)

Aqui trataremos das intoxicações exógenas que são um sério problema de saúde pública, constituindo uma das principais causas de atendimento em emergências. Caracterizada por desequilíbrio fisiológico, consequente das alterações bioquímicas no organismo em que é evidenciado por sinais e sintomas ou mediante dados laboratoriais. A Toxicologia é a ciência que estuda os efeitos adversos decorrentes das interações de substâncias químicas com o organismo, a substância nesse caso é chamada de toxicantee a consequência de sua ação é definida como intoxicação. Toda substância possui um potencial tóxico, que depende da própria atividade química da espécie viva envolvida, da genética, da exposição (contato), da via de ingestão ou inoculação e do próprio organismo (OGA, 2008)

As substâncias químicas podem ser encontradas tanto no ambiente como ar, água, alimentos, plantas, animais peçonhentos ou venenosos, ou isolados como os pesticidas, medicamentos, produtos de uso industrial, produtos de uso domiciliar, entre outros. Essas intoxicações podem ser acidentais ou intencionais e são importantes causas de agravos à saúde, sendo um problema de saúde pública mundial, pois de acordo com aOMS 
(OMS, 2014) quase um milhão de pessoas morrem a cada ano.

De acordo com os centros de informação e assistência toxicológica distribuídos pelo país, os medicamentos, animais peçonhentos e produtos domissanitários, são os principais agentes causadores de intoxicações em seres humanos, entretanto, existem outros produtos que provocam ocorrência de intoxicações, como por exemplo, os agrotóxicos.O Brasil é considerado o campeão mundial em consumo de agrotóxicos. Em 2008, o país utilizou 673.862 toneladas de agrotóxicos. O Paraná utiliza 12 quilos de agrotóxico por hectare ao ano, enquanto a média brasileira de consumo é de 4 quilos/ha/ano. Os agrotóxicos utilizados no estado são considerados "muito perigosos" e "perigosos", numa classificação que vai de "pouco" a "altamente perigoso (IBGE, 2012)

$\mathrm{Na}$ maioria dos municípios, a vigilância sanitária em conjunto com a vigilância epidemiológica é responsável pelos dados das intoxicações, utilizando-se o Sistema de Informação de Agravos de Notificação - SINAN que está implantado em todo o país, é um sistema mais recente, complexo, gerenciado dentro da Secretaria Nacional de Vigilância a Saúde - SNVS, foi desenvolvido no início da década de 90, tendo como objetivo a coleta e processamento dos dados sobre agravos de notificação em todo o território nacional(FREITAS et al., 2012)
Estudos sobre intoxicação exógena sob o ponto de vista da vigilância sanitária são relevantes, para mostrar a dimensão do problema nos municípios e apontar caminhos para a ação dos serviços de vigilância sanitária, de monitoramento dessas substâncias, criação de bancos de dados com informações e medidas de controle da sociedade para intervenção nos danos causados (SILVA, 2009)

No mundo é relatado cerca de três milhões de vítimas e mais de 220 mil mortes causadas anualmente por intoxicações, considerada importante causa de morbidade e mortalidade. Nesse sentido faz-se necessário identificar os casos decorrentes por intoxicações exógenas no município de Santarém Pará, assim como os principais problemas referentes a qualquer indivíduo que pode estar suscetível a riscos graves e ao óbito pela grande capacidade tóxica das substâncias, que através desta pesquisa poderá propor soluções que ajudem a minimizar a problemática.

\section{METODOLOGIA DA PESQUISA}

\section{Técnica de coleta e análise dos dados}

A pesquisa desenvolveu-se a partir dos dados fornecidos no setor do Centro de Controle de Zoonoses (CCZ) que é uma unidade de saúde pública que têm como atribuição fundamental de prevenir e controlar as zoonoses (como raiva e o calazar, além da 
dengue e doença de chagas), este desenvolve sistemas de vigilância sanitária e epidemiológica, e direciona os dados dos casos e óbitos, que são encaminhados dos estabelecimentos de saúde para o CCZ, através de fichas de investigação do SINAN, em, que faz notificação compulsória Portaria GM/MS N ${ }^{\circ}$ 104, DE 25 DE JANEIRO DE 2011), tem por objetivo a coleta e processamento dos dados sobre agravos de notificação em todo o território nacional, fornecendo informações para a análise do perfil da morbidade e contribuindo, dessa forma, para a tomada de decisões nos níveis municipal, estadual e federal.

A identificação dos casos foi realizada pela leitura das fichas, das quais foram analisadas as seguintes variáveis: faixa etária, sexo, frequência por agente tóxico, tipo de atendimento, critério de confirmação e evolução. Os dados foram coletados por meio de planilha eletrônica do software Excel versão Microsoft Windows XP que é um aplicativo informatizado que realiza leitura e tabulação. Foram cumpridos os princípios éticos estabelecidos pela resolução 466/12, do ministério da saúde.

\section{RESULTADOS E DISCUSSÃO}

Nos anos de 2009 a 2013, foram registrados em Santarém, a totalidade de 269 casos de intoxicações causadas por agentes químicos exógenos, sendo que 33 casos foram classificados como ignorado/branco. Os casos considerados Ignorado/branco se referem a situações em que a vigilância Epidemiologia não conseguiu, por circunstâncias variadas, definir o tipo de agente envolvido na intoxicação exógena, conforme tabela 2.

Na tabela 1 é possível observar que a faixa etária de 20 a 39 ocorreram os maiores índices de intoxicações com 34,9\% (94/269) dos casos, observando estudos na literatura pode-se perceber que a maioria das cidades brasileiras também ocorreram o predomínio de casos de intoxicação nessa faixa etária, como por exemplo, um estudo realizado por Pedro e Marcella (2013) na mesorregião norte central paranaense, em que os resultado prevaleceram da mesma forma.

Na faixa etária de 10 a 19 anos com $22,3 \%(60 / 269)$ e a faixa etária de 1 a 4 anos de idade com 22,3\% (60/269) dos casos, ambos ocupando o segundo lugar nos casos de intoxicação. De acordo com Werneck eHasselmann (2009) as crianças menores de cinco anos de idade formam um grupo vulnerável às intoxicações acidentais, principalmente pela curiosidade, elas exploram o ambiente com todos os sentidos, o que favorece o contato e a ingestão de agentes tóxicos, a maioria dessas intoxicações ocorrem no próprio local de moradia sendo que as substâncias envolvidas não estão adequadamente armazenadas. 
Tabela 1 - FREQUÊNCIA POR FAIXA ETÁRIA, SEGUNDO ANO DE NOTIFICAÇÃO.

\begin{tabular}{|c|c|c|c|c|c|c|c|c|c|c|c|}
\hline \multirow[t]{2}{*}{ Faixa Etária } & \multicolumn{2}{|c|}{2009} & \multicolumn{2}{|c|}{2010} & \multicolumn{2}{|c|}{2011} & \multicolumn{2}{|c|}{2012} & \multicolumn{2}{|c|}{2013} & \multirow{2}{*}{$\begin{array}{c}\text { Total } \\
\mathbf{N} \\
\end{array}$} \\
\hline & $\mathbf{N}$ & $\%$ & $\mathbf{N}$ & $\%$ & $\mathbf{N}$ & $\%$ & $\mathbf{N}$ & $\%$ & $\mathbf{N}$ & $\%$ & \\
\hline$<1$ ano & 0 & 0 & 0 & 0 & 0 & 0 & 3 & 50 & 3 & 50 & 6 \\
\hline $1-4$ anos & 3 & 5 & 1 & 2 & 19 & 32 & 15 & 25 & 22 & 36 & 60 \\
\hline 5 - 9 anos & 0 & 0 & 1 & 7,6 & 3 & 23 & 5 & 38 & 4 & 31 & 13 \\
\hline $10-19$ anos & 4 & 7 & 4 & 7 & 18 & 30 & 15 & 23 & 20 & 33 & 60 \\
\hline 20 - 39 anos & 3 & 3 & 7 & 7 & 31 & 33 & 26 & 28 & 27 & 29 & 94 \\
\hline 40 - 59 anos & 3 & 13 & 1 & 5 & 10 & 43 & 3 & 13 & 6 & 26 & 23 \\
\hline $60 \mathrm{e}+\operatorname{anos}$ & 1 & 8 & 2 & 15 & 4 & 31 & 4 & 38 & 1 & 8 & 13 \\
\hline Total & 14 & 100 & 16 & 100 & 85 & 100 & 71 & 100 & 83 & 100 & 269 \\
\hline
\end{tabular}

Fonte: SINAN, Santarém 2009 a 2013.

As intoxicações foram predominantes em indivíduos do sexo feminino, com 59,1\% (159/269), em todos os anos, com exceção do ano de 2009, em que ocorreu a predominância do sexo masculino, conforme mostrado no gráfico 1. Bernardes et al (2010) realizou um estudo sobre tentativas de suicídio e suicídio consumado na cidade de Ribeirão Preto-SP, concluindo que, nas tentativas de suicídio, as mulheres apresentam uma frequência duas vezes mais elevada em relação aos homens, através da ingestão de medicamentos e outras substâncias químicas, e, nos suicídios consumados, a frequência é de 2,8 homens para uma mulher. Em outro estudo realizado por Zambolim (2013), afirma que os homens são mais suscetíveis ao suicídio, tentam menos frequentemente, mas com métodos mais eficazes, por outro lado, as mulheres tentam mais frequentemente e têm menos êxito, por visarem a drogas menos tóxicas. 
Gráfico 1 - FREQUÊNCIA POR GÊNERO, SEGUNDO ANO DE NOTIFICAÇÃO.

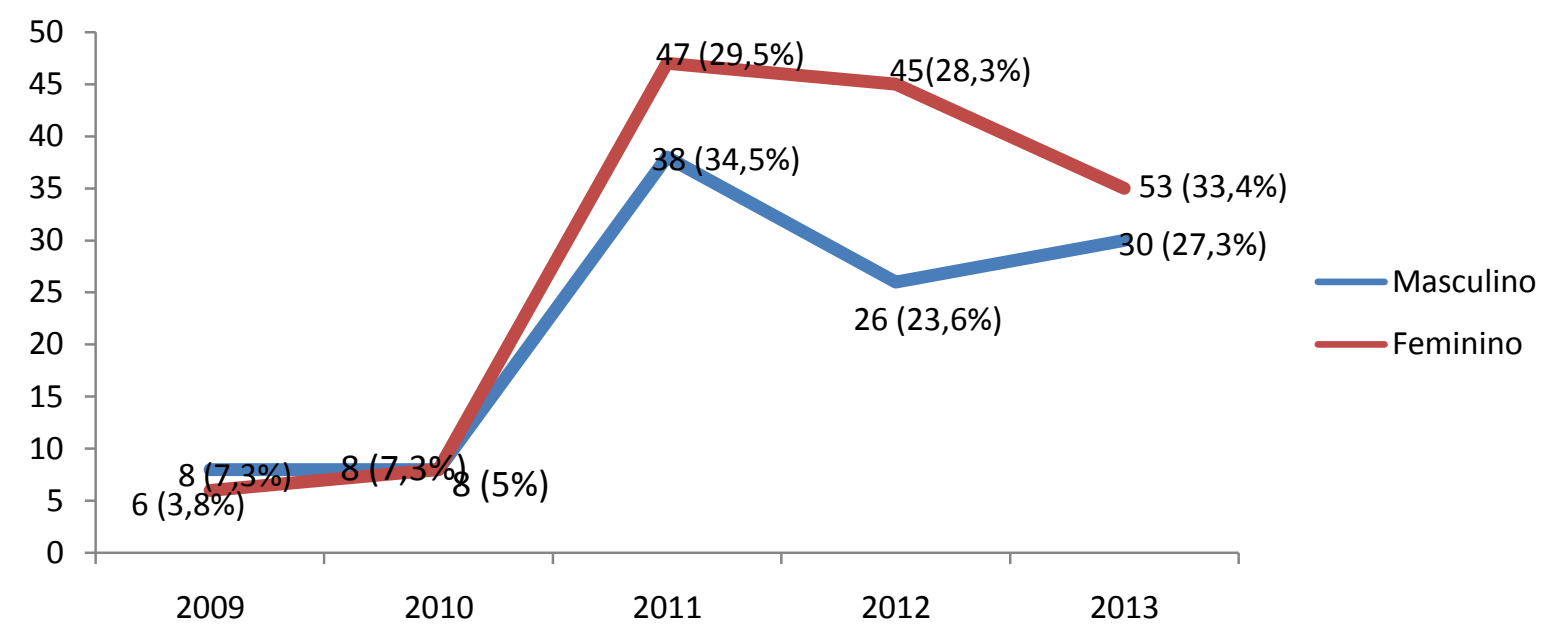

Fonte: SINAN, Santarém 2009 a 2013.

Das 269 notificações registradas pelo SINAN no município de Santarém nos anos de 2009 a 2013 os principais responsáveis pelas intoxicações foram os medicamentos, correspondendo a $62(23,1 \%)$ casos, conforme tabela 2. Segundo a ANVISA (2009), 18\% das mortes por envenenamento se devem à automedicação e $23 \%$ dos casos de intoxicação infantil estão ligados à ingestão acidental de medicamentos armazenados de forma incorreta.

De acordo com o SINITOX (2008), a intoxicação por medicamento se deve por vários fatores, como as técnicas de marketing que atraem tanto prescritores quanto os usuários de medicamentos e isso favorece a utilização inadequada desses produtos, assim como a utilização abusiva de embalagens atraentes, medicamentos coloridos e

adocicados, com sabor de frutas e formato de bichinhos que colaboram para o aumento das intoxicações acidentais em crianças.

A segunda maior causa de intoxicação foi por raticida, e correspondeu cerca de 19,7\% (53/269) dos casos. De acordo com a ANVISA (2009) o raticida utilizado frequentemente nas intoxicações é conhecido popularmente como "chumbinho", as estimativas do governo apontam que $o$ produto é responsável por quase $60 \%$ dos 8 mil casos de intoxicação relacionados a "chumbinho" no Brasil todos os anos.

No ano de 2011 os principais agentes responsáveis por intoxicação exógena foram os alimentos e bebidas com 11,5\% (31/269). Neste ano no município de Santarém ocorreu um surto de intoxicação alimentar envolvendo crianças de uma escola da rede municipal de ensino(SINITOX, 2008). 
Tabela 2 - FREQUÊNCIA POR AGENTE TÓXICO.

\begin{tabular}{|c|c|c|c|c|c|c|c|c|c|c|c|}
\hline \multirow[t]{2}{*}{ Agente Tóxico } & \multicolumn{2}{|c|}{2009} & \multicolumn{2}{|c|}{2010} & \multicolumn{2}{|c|}{2011} & \multicolumn{2}{|c|}{2012} & \multicolumn{2}{|c|}{2013} & \multirow{2}{*}{$\begin{array}{c}\text { Tota } \\
\mathbf{l}\end{array}$} \\
\hline & $\mathbf{N}$ & $\%$ & $\mathbf{N}$ & $\%$ & $\mathbf{N}$ & $\%$ & $\mathbf{N}$ & $\%$ & $\mathbf{N}$ & $\%$ & \\
\hline $\begin{array}{l}\text { Ignorado/ } \\
\text { Branco }\end{array}$ & 6 & 18,2 & 2 & 6,1 & 5 & 15,1 & 9 & 27,3 & 11 & 33,3 & 33 \\
\hline Medicamento & 1 & 1,6 & 5 & 8,1 & 17 & 27,4 & 15 & 24,2 & 24 & 38,7 & 62 \\
\hline $\begin{array}{l}\text { Agrotóxico } \\
\text { agrícola }\end{array}$ & 0 & 0 & 0 & 0 & 4 & 20 & 12 & 60 & 4 & 20 & 20 \\
\hline $\begin{array}{l}\text { Agrotóxico } \\
\text { doméstico }\end{array}$ & 0 & 0 & 0 & 0 & 3 & 17 & 8 & 44 & 7 & 39 & 18 \\
\hline $\begin{array}{l}\text { Agrotóxico } \\
\text { saúde pública }\end{array}$ & 0 & 0 & 0 & 0 & 0 & 0 & 1 & 100 & 0 & 0 & 1 \\
\hline Raticida & 1 & 9 & 5 & 9 & 18 & 34 & 14 & 27 & 11 & 21 & 53 \\
\hline $\begin{array}{l}\text { Produto } \\
\text { Veterinário }\end{array}$ & 0 & 0 & 2 & 29 & 0 & 0 & 3 & 42 & 2 & 29 & 7 \\
\hline $\begin{array}{l}\text { Produto } \\
\text { domiciliar }\end{array}$ & 0 & 0 & 1 & 9 & 0 & 0 & 2 & 18 & 8 & 73 & 11 \\
\hline Cosméticos & 0 & 0 & 0 & 0 & 0 & 0 & 1 & 50 & 1 & 50 & 2 \\
\hline $\begin{array}{l}\text { Produtos } \\
\text { Químicos }\end{array}$ & 0 & 0 & 1 & 7 & 10 & 66 & 3 & 20 & 1 & 7 & 15 \\
\hline $\begin{array}{l}\text { Alimentos e } \\
\text { bebidas }\end{array}$ & 1 & 3 & 0 & 0 & 19 & 62 & 3 & 9 & 8 & 26 & 31 \\
\hline Planta tóxica & 0 & 0 & 0 & 0 & 2 & 100 & 0 & 0 & 0 & 0 & 2 \\
\hline Drogas de abuso & 0 & 0 & 0 & 0 & 0 & 0 & 0 & 0 & 1 & 0 & 1 \\
\hline Outros & 1 & 7 & 0 & 0 & 7 & 54 & 0 & 0 & 5 & 39 & 13 \\
\hline Total & 14 & 100 & 16 & 100 & 85 & 100 & 71 & 100 & 83 & 100 & 269 \\
\hline
\end{tabular}

Fonte: SINAN, Santarém 2009 a 2013.

A zona urbana concentra a maioria dos casos de intoxicação no município de Santarém, em todos os anos analisados. Em uma pesquisa realizada no sul do Brasil na cidade de MaringáeParaná mostrou que a zona urbana concentra a maioria das intoxicações e o principal agente tóxico são os 
medicamentos (MARGONATO et al., 2009). Em contrapartida, apesar de a legislação brasileira ser bastante moderna e abrangente, os casos de intoxicações em trabalhadores rurais são frequentes no país de modo geral. A maior probabilidade de ocorrências de intoxicações na zona rural foi encontrado entre os trabalhadores, assalariado ou temporário, decorrente da exposição pelo agente agrotóxico(SINITOX, 2008)

O tipo de intoxicação que predominou nestes anos no município foi à aguda, $52 \%$ dos casos. Segundo Rebelo (2011), no Brasil os atendimentos registrados são, majoritariamente, relacionados aos casos de intoxicação aguda.

O critério clínico-epidemiológico representou 48,69\% (131/269) dos casos seguido do critério clinico com 29,7\% (80/269). O clinico laboratorial representou $13,38 \%$ (36/269) casos. Segundo OGA
(2008), o diagnóstico laboratorial, se faz necessário principalmente nas intoxicações por agrotóxicos agrícolas para se determinar o tratamento adequado e administração dos antídotos específicos, pois, uma intoxicação é um processo que ocorre após a exposição do indivíduo a um agente tóxico, que é evidenciado por sinais e sintomas, ou mediante dados laboratoriais.

A cura sem sequela representou o tipo de evolução mais frequente, com $66,9 \%$ $(180 / 269)$ dos casos notificados. Esse percentual indica de forma geral que os atendimentos hospitalares estão respondendo as ocorrências satisfatoriamente, mesmo sem serviço especializado. Nos anos da referida pesquisa ocorreram 3 óbitos, conforme gráfico 2 .

\section{Gráfico 2 - FREQUÊNCIA POR EVOLUÇÃO.}

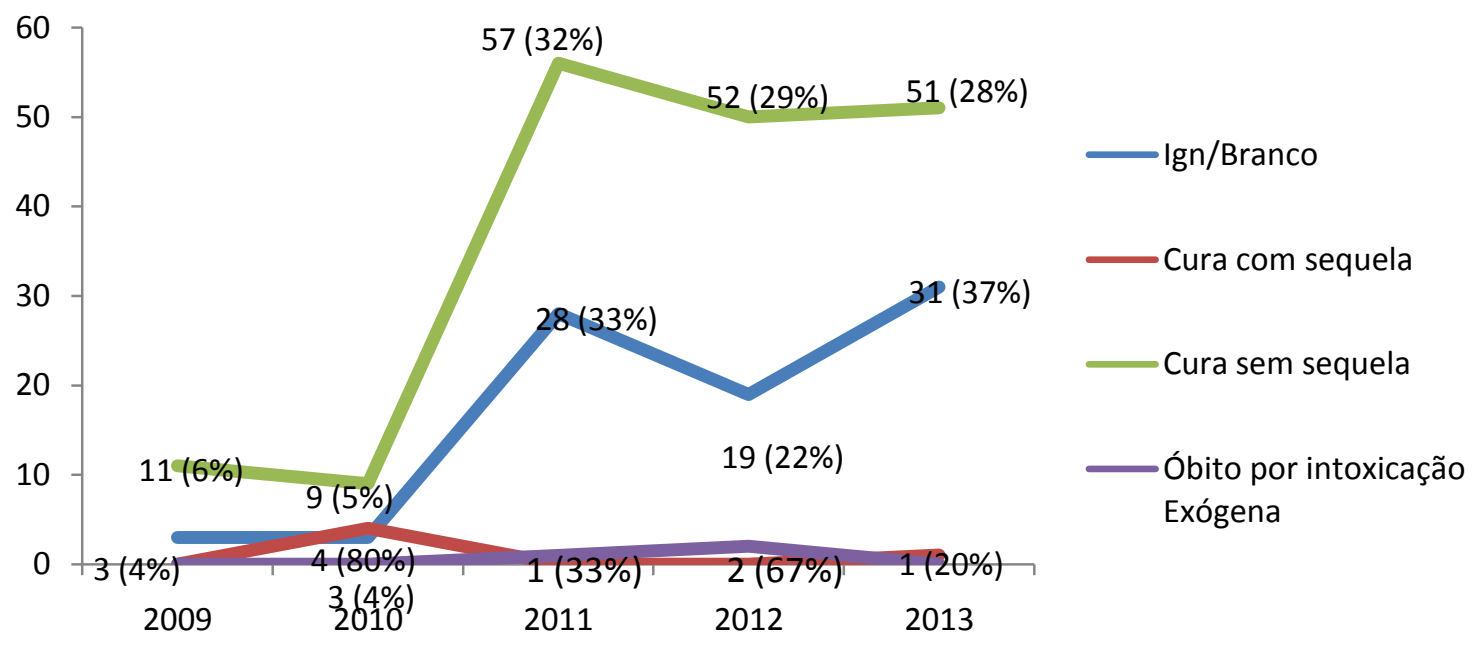

Fonte: SINAN, Santarém 2009 a 2013. 


\section{CONSIDERAÇÕES FINAIS}

Neste estudo onde possibilitou identificar o perfil das intoxicações exógenas em Santarém-Pará, no período de 2009 a 2013 a partir de dados do sistema de informações de agravos de notificação (SINAN), foi constatado que o maior índice de intoxicações foi por medicamentos, seguido de raticida predominando no sexo feminino. De modo geral, os resultados obtidos trazem evidências claras do impacto na saúde humana produzidos por essas substâncias.

O medicamento ocupa papel fundamental na busca pela recuperação da saúde e é elemento essencial das práticas profissionais. Cabe ao profissional farmacêutico orientar e informar a população sobre uso correto e racional dos medicamentos, que os mesmos trazem riscos à saúde se usados de forma incorreta, podendo, desta forma, causar reações

\section{REFERÊNCIAS}

ANVISA. Agência Nacional de Vigilância Sanitária. 2009. Disponível em: $<$ http:। www.portal.anvisa.gov.br $>$, acesso em 23/03/2014

BERNARDES, Sara Santos; TURINI, Conceição Aparecida and MATSUO, Tiemi. Perfil das tentativas de suicídio por sobredose intencional de medicamentos atendidas por um Centro de Controle de Intoxicações do Paraná, Brasil. Cad. Saúde Pública. 26 (7), 2010. indesejadas ou intoxicação pelo uso indiscriminado. Portanto, observamos que há uma grande necessidade de se fazer campanhas educativas, por parte do governo, com objetivo de ampliar o conhecimento da população de Santarém acerca dos cuidados em relação aos medicamentos e substâncias químicas, ajudando a reduzir o impacto destas ocorrências no município. Fica a necessidade de mais estudos desta ordem para que se possa acompanhar o perfil epidemiológico das intoxicações exógenas no município de Santarém-Pará, com o intuito de estabelecer estratégias que previnam o surgimento de novos casos e de reincidências.

FILHO, Janilson da Silva. Intoxicações exógenas no município de Sobral-Ceará, sob a ótica da vigilância sanitária, 2009. Disponível em; http: // www.esp.ce.gov.br >acesso em: 18/01/2014.

FREITAS, Ronilson Ferreira; Royo, Vanessa de Andrade; Moura,Paula Maria Soares de.

Avaliação do risco de intoxicações por medicamentos, domissanitários e agrotóxicos na população de juramento MG. Conexão ciência, 7(1), 2012. 
HASSELMANN, Maria Helena et al. Intoxicações exógenas em crianças menores de 6 anos atendidas em hospitais da região metropolitana do Rio de Janeiro. Rev.

Assoc. Med. Bras. 55(3), 2009.

Instituto Brasileiro de Geografia e Estatística (IBGE). Indicadores de Desenvolvimento

Sustentável: Brasil 2012. Estudos e

Pesquisas. Informação Geográfica, n. 9. Rio de Janeiro: IBGE; 2012.

MANGOLINI, Pedro Dias Neves;

BELLINI,Marcella. Intoxicações por agrotóxicos na mesorregião norte central paranaense, Brasil - 2002 a 2011.Ciênc. saúde coletiva. vol.18 no.11 Rio de Janeiro nov. 2013

MARGONATO, Fabiana Burdini; THOMSON, Zuleikaand PAOLIELLO, Mônica Maria Bastos. Determinantes nas intoxicações medicamentosas agudas na zona urbana de um município do Sul do Brasil.Cad. Saúde Pública, 24 (2), 2008.

OGA, Zeigiet al. Fundamentos de toxicologia. $3^{\circ}$ edição. São Paulo. Atheneu editora, 2008.

OMS, Organização Mundial de Saúde. Ministério da saúde manual de vigilância de saúde de populações expostas a agrotóxicos, 2010. Disponível em: <http: www.spb.ufsc.br >.acesso em: 12/03/2014.

REBELO, Fernanda Maciel et al. Intoxicação por agrotóxicos no Distrito Federal, Brasil, de 2004 a 2007 - análise da notificação ao

Centro de Informação e Assistência

Toxicológica . Ciênc. saúde coletiva, Ago

2011, vol.16, no.8,

SINITOX. Sistema Nacional de Informações Tóxico farmacológicas, 2008. Disponível em: <http: www.fiocruz.br/sinitox >acesso em: 09/02/2014.

ZAMBOLIM, Cristiane Maciel et al. Perfil das intoxicações exógenas em um hospital universitário, Rev. méd. Minas Gerais, 18 (1), 2013. 